\title{
Eccrine Spiradenoma of the Scalp
}

\author{
Jaehee Kim ${ }^{1}$, \\ Ho Jik Yang ${ }^{1}$, \\ Jung-Soo Pyo ${ }^{2}$ \\ Departments of ${ }^{1}$ Plastic and Reconstructive \\ Surgery and ${ }^{2}$ Pathology, Eulji University \\ College of Medicine, Daejeon, Korea
}

No potential conflict of interest relevant to this article was reported.
Eccrine spiradenoma is an uncommon benign adnexal tumor originating from the eccrine sweat gland. We diagnosed a eccrine spiradenoma on a 55-year-old man with histopathologic confirmation upon biopsy followed by complete resection, who had visited our clinic with a chief complain of occipital scalp mass. The solitary eccrine spiradenoma occurring in the scalp is rarely to be seen and should be considered as a differential diagnosis for a solitary cystic mass of the scalp.

Keywords: Eccrine spiroadenoma / Scalp mass / Benign adnexal tumor

\section{INTRODUCTION}

Eccrine spiradenoma is an uncommon benign tumor of skin adnexa originating from eccrine glands, which was first described by Kersting and Helwig [1]. It is usually appeared as a single nodule, but also appears rarely as multiple nodules, and being distributed as a linear form or zosteriform [2]. The incidence rate is not difference according to one's racial or gender differences and it occurs in young adults of $15-35$ years usually, but rarely occurs in infants as well [3]. It can occur mainly in the head, neck, and trunk and sometimes accompany with pain and tenderness [4]. The occurrence of eccrine spiradenoma as a single lesion on the scalp is rare. Thus we report a rare case of eccrine spiradenoma appeared on the scalp region as a solitary lesion along with reference checking and histologic findings.

\section{CASE REPORT}

A 55-year-old man presented with a mass on the scalp of occipital region with unknown onset time. There were no specific findings

Correspondence: Ho Jik Yang

Department of Plastic and Reconstructive Surgery, Eulji University Hospital, 95

Dunsanseo-ro, Seo-gu, Daejeon 35233, Korea

E-mail: drhjyang@eulji.ac.kr

Received April 11, 2017 / Revised September 15, 2017 / Accepted September 15, 2017 in the past and family history. The surface of the mass was smooth and light pink- colored containing some hairs within. The mass was approximately $1.2 \times 1.2 \mathrm{~cm}$ in size, conical in shape and firmly fixed to the scalp and there were no changes or any secretions visible around the scalp lesion (Fig. 1). All tumors were undergone complete resection with $2 \mathrm{~mm}$ safety margin and biopsied afterward for accurate diagnosis and treatment (Fig. 2). The patient was being laid prone position on the operating table for the removal of tumors and primary sutures were performed under local anesthesia without any visible defect left on the scalp. Upon histopathologic examination, the resected nodules were surrounded by a well-defined membrane with high cell density and being arranged by epithelial cells in coded forms (Fig. 3A).

Two types of distinctive cells were clearly visible: firstly, large central cuboidal cells containing eosinophilic cytoplasm with follicular nuclei and secondly, peripheral small cells containing dark nucleus and pale cytoplasm (Fig. 3B). Thus the diagnosis of an eccrine spiradenoma was made according to the findings provided. There was no recurrence and the biopsy site was well maintained without any healing defect on 6 months follow-up.

\section{DISCUSSION}

Eccrine spiradenoma is a rare benign tumor of skin adnexa being 


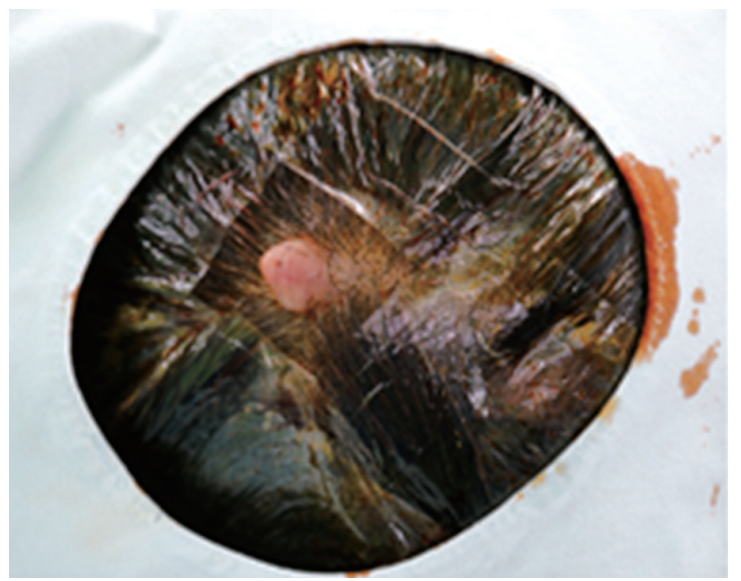

Fig. 1. Showing the tumor, preoperative photograph. A 55-year-old male presented with a solitary mass on the scalp.

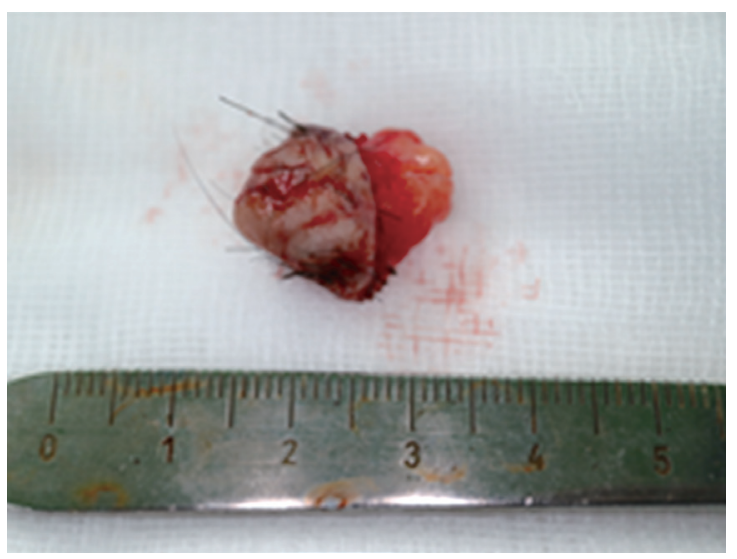

Fig. 2. Showing the resected tumor mass, intraoperative photograph. The resected specimen measured approximately $1.2 \times 1.2 \times 1.8 \mathrm{~cm}$.

presented as a bluish/pinkish cystic tumor under the skin and subcutaneous tissue with varying size of 0.3 to $5 \mathrm{~cm}$, open accompanying with mild tenderness. The eccrine spiradenoma, if occurs solitarily, can occur in various parts of the body usually in the front of the body and above the waistline [1]. However, it is rarely to be seen as a solitary lesion occurring from the scalp region like in this case.

Clinically, it is difficult to differentiate eccrine spiradenoma from others, therefore biopsy is vital for accurate diagnosis. It is important to find out epithelial cells, myoepithelial cells and lymphocytes along with void spaces between blood vessels under the microscopes for diagnostic confirmations. Two distinctive cells
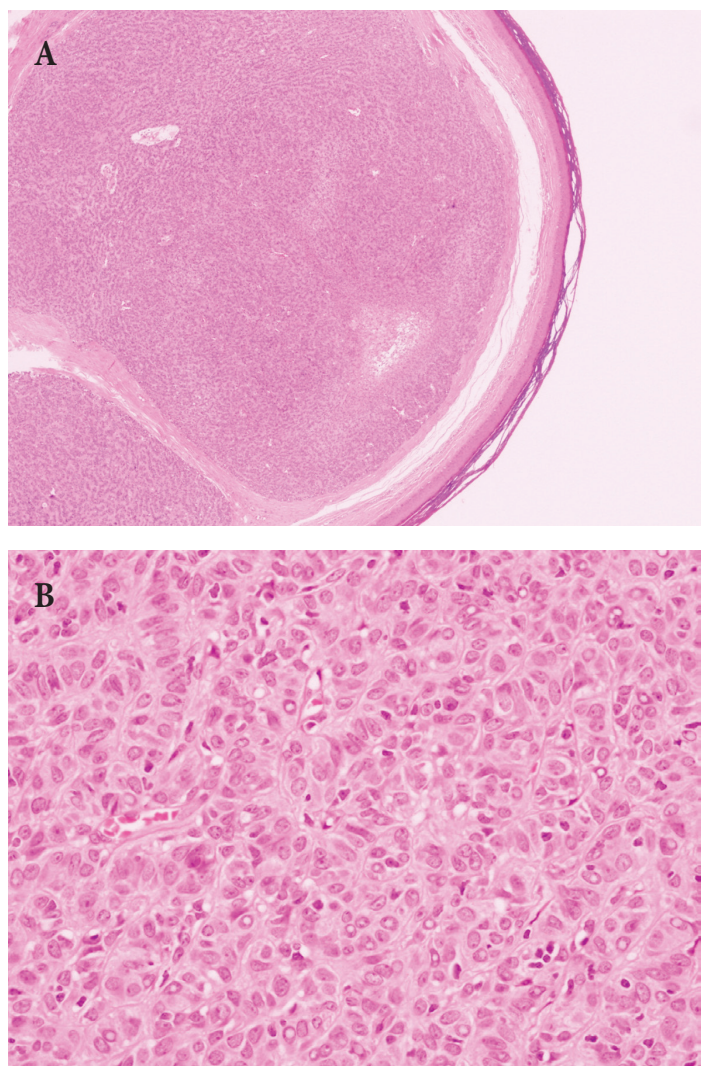

Fig. 3. Microscopic findings of the tumor. (A) Well-circumscribed and encapsulated tumor nodule arising from the dermis (H\&E, $\times 40)$. (B) Tumor lobules composed of two cell types. Peripherally, smaller dark blue cells and centrally, pale larger cells. Large cells showing luminal differentiation containing eosinophilic material at the center $(\mathrm{H} \& \mathrm{E}, \times 200)$.

can also be observed. Cells with large nucleus and pale cytoplasm are located in the center, and cells with smaller dense nucleus are located in the periphery. The lumen of this contains an acidic substance which is being arranged around a smaller lumen. Eccrine spiradenoma may accompany with similar tumors of apocrine origin, such as trichoblastoma and cylindroma. Histologically, the Eccrine glands have relatively clear cytoplasm and occasionally squamous epithelium whereas the apocrine glands have eosinophilic cytoplasm and nuclei located at the base bottom (off centered) [5].

Eccrine spiradenoma should be considered in the differential diagnosis of skin tumors associated with epidermal ulceration and multiple skin tumors whether grouped together or far removed from each other. In addition, eccrine spiradenoma had a 
diagnostic problem as same typical features of dermal cylindroma $[1,6]$. Dermal cyclindroma, however, can be differentiated histologically from eccrine spiradenoma. Comprised of cords of cells, surrounded by a prominent basement membrane, that exhibit a mosaic pattern, they lack pseudoglandular structures and a true capsule, have more hyaline material and are more superficial with a tendency to approach the epidermis which bay be flattened or atrophic [7].

Although a few cases of malignant change from a part of the eccrine spiradenoma were reported, but this happens very rarely indeed. Malignant changes may occur if the eccrine spiradenoma lasts long enough, clinically by showing rapidly increased size of cystic lesion. Malignant transformation of eccrine spiradenoma shares many clinical and pathologic features with malignant transformation of dermal cylindroma. As in malignant spiradenoma, the carcinomas that develop in cylindroma often retain architectural features evocative of cylindroma, including a pattern of closely spaced, cellular nests and trabeculae, a basaloid cell type, and remnants of hyaline membranes about some tumor nests [8].

It is difficult to determine the precise treatment guidelines for the eccrine spiradenoma on the scalp because of lacking of cases treated, but this tumor does not seem to recur if complete resection had been pursued.

Therefore, early diagnosis and complete resection of the ec- crine spiradenoma of scalp is considered to be an appropriate guideline of treatment, as some sweat gland malignancies have a poor prognosis.

In conclusion, the eccrine spiradenoma is a rarely occurring scalp tumor and should be considered as a differential diagnosis for the solitary cystic tumor around the scalp area and also a complete resection of the tumor should be considered as a preferable treatment option.

\section{REFERENCES}

1. Kersting DW, Helwig EB. Eccrine spiradenoma. AMA Arch Derm 1956;73:199-227.

2. Wright S, Ryan J. Multiple familial eccrine spiradenoma with cylindroma. Acta Derm Venereol 1990;70:79-82.

3. Ekmekci TR, Koslu A, Sakiz D. Congenital blaschkoid eccrine spiradenoma on the face. Eur J Dermatol 2005;15:73-4.

4. Scheinfeld NS, Tarlow MM, Burgin S. Blaschkoid eccrine spiradenomas. Cutis 2002;70:73-5.

5. Kim MH, Cho E, Lee JD, Cho SH. Giant vascular eccrine spiradenoma. Ann Dermatol 2011;23:S197-200.

6. Mambo NC. Eccrine spiradenoma: clinical and pathologic study of 49 tumors. JCutan Pathol 1983;10:312-20.

7. Crain RC, Helwig EB. Dermal cylindroma (dermal eccrine cylindroma). Am J Clin Pathol 1961;35:504-15.

8. Cooper PH, Frierson HF Jr, Morrison AG. Malignant transformation of eccrine spiradenoma. Arch Dermatol 1985;121:1445-8. 